\title{
Enhanced efficiency and reduced spectral shift of green light-emitting-diode epitaxial structure with prestrained growth
}

\author{
Chi-Feng Huang, Tzu-Chi Liu, Yen-Cheng Lu, Wen-Yu Shiao, Yung-Sheng Chen, \\ Jyun-Kai Wang, Chih-Feng Lu, and C. C. Yang ${ }^{a}$ \\ Institute of Photonics and Optoelectronics and Department of Electrical Engineering, \\ National Taiwan University, 1, Roosevelt Road, Section 4, Taipei 10617, Taiwan
}

(Received 1 July 2008; accepted 5 November 2008; published online 18 December 2008)

\begin{abstract}
The enhanced emission efficiency and reduced spectral shifts of a green InGaN/GaN quantum-well (QW) light-emitting-diode epitaxial structure by using the prestrained growth technique when compared with a control sample of the same emission spectrum with conventional growth are demonstrated. By adding an $\sim 7 \%$-indium InGaN/GaN QW to the structure before the growth of designated emitting high-indium QWs, the growth temperature of the emitting QWs can be raised by $30{ }^{\circ} \mathrm{C}$ while keeping about the same emission wavelength around $544 \mathrm{~nm}$ in photoluminescence $(\mathrm{PL})$ and $525 \mathrm{~nm}$ in electroluminescence (EL). The internal quantum efficiency, room-temperature PL intensity, and EL intensity at the injection current of $20 \mathrm{~mA}$ are increased by $\sim 167 \%, \sim 140 \%$, and $\sim 182 \%$, respectively. Also, the spectral blueshift range in increasing injection current in the range of $50 \mathrm{~mA}$ is decreased by $46 \%$. Based on the pump-power dependent PL measurement, it is found that the quantum-confined Stark effect (QCSE) becomes weaker in the prestrained growth sample. Also, from the calibration of the Arrhenius plots, the carrier localization effect is observed to become weaker under prestrained growth. Therefore, the enhanced emission efficiency is mainly attributed to the decreased defect density and the reduced QCSE in the prestrained sample. (C) 2008 American Institute of Physics. [DOI: 10.1063/1.3046582]
\end{abstract}

\section{INTRODUCTION}

In developing the technology of light-emitting diode (LED) for display and lighting applications, green light generation still represents one of the major problems. Although the quaternary compound of $\mathrm{AlGaInP}$ has been widely used for fabricating red LED, its indirect band gap nature in the green range leads to inefficient emission. ${ }^{1}$ On the other hand, in using InGaN/GaN quantum wells (QWs) for emitting green light, the indium content needs to be higher than $15 \%$. With such high indium content, spinodal decomposition in the QW results in composition fluctuation and the formation of indium-rich nanoclusters. ${ }^{2}$ Although indium-rich nanoclusters can lead to carrier localization and hence radiative recombination enhancement in the green range, ${ }^{3-5}$ they are usually accompanied by nearby stacking faults. ${ }^{6}$ Therefore, normally the internal quantum efficiency (IQE) drops to $<10 \%$ when the indium content of QW is increased to a certain level for emitting green light. Recently, efforts were made to improve the efficiency of green LED by using relatively lower-temperature grown $\mathrm{InGaN}$ for $p$-type layer through the QW strain relaxation and the reduction in thermal annealing effect in QWs during the high-temperature $p$-type $\mathrm{GaN}$ growth. ${ }^{7,8}$

In this paper, we demonstrate the enhanced IQE of a green-emitting InGaN/GaN QW based on the prestrained growth technique. ${ }^{9-11}$ In this metalorganic chemical vapor deposition (MOCVD) technique, a low-indium InGaN layer is deposited before the growth of high-indium $\mathrm{InGaN} / \mathrm{GaN}$ QWs for creating a tensile strain in the GaN barrier layer

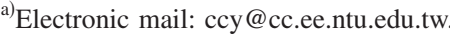

right above it. The indium content and thickness of the lowindium InGaN layer need to be well adjusted such that the possibly strongest tensile strain can be generated in the $\mathrm{GaN}$ barrier layer. Higher indium content can lead to a larger lattice mismatch and hence stronger strain. However, when the indium content is larger than a certain level, spinodal decomposition can occur to relax the strain within the InGaN layer. ${ }^{12,13}$ With the compressive strain preserved in the InGaN layer of suitable indium content, it is expected to produce a local perturbation in the global strain distribution, which is controlled by the thick GaN layer below the lowindium InGaN layer. In this situation, a tensile strain is built in the thin $\mathrm{GaN}$ barrier layer above it. Based on our tests, $6 \%-7 \%$ indium content in a $3 \mathrm{~nm} \mathrm{InGaN} / \mathrm{GaN}$ QW represents an optimized condition for creating a significant tensile strain in the GaN barrier. Such a tensile strain, even though it is quite small, can result in significantly enhanced incorporation of larger-size indium atoms in growing the subsequent high-indium InGaN/GaN QWs, leading to higher indium content at a particular growth temperature. With the prestrained growth technique, high-indium QWs for fabricating orange-red and white-light LEDs have been implemented. ${ }^{14,15}$ In this paper, we compare four LED epitaxial samples to show the improved emission efficiency of green-emitting QWs by using the prestrained growth technique. Electroluminescence (EL) and temperature-dependent photoluminescence (PL) measurements show the relatively stronger green emission intensity of the prestrained sample when compared with a control sample of about the same emission wavelength. Also, power dependent PL and injection-current dependent EL measurements show the 
smaller emission wavelength shifts compared to those of the control sample. Time-resolved PL (TRPL) measurement, analysis based on Arrhenius plot, and transmission electron microscopy (TEM) are performed to explain the aforementioned enhanced emission efficiency and smaller-wavelength shifts. In Sec. II of this paper, the sample structures and the conditions for sample preparation are presented. The x-ray diffraction (XRD) measurement results are also shown in this section. The PL and EL measurement data are reported in Sec. III. Then, discussions are made in Sec. IV, in which other measurement results are used to explain what are observed in Sec. III. Finally, conclusions are drawn in Sec. V.

\section{SAMPLE STRUCTURES AND PREPARATION CONDITIONS}

Four samples of LED structures were prepared for comparison. Among the four samples, which were grown on $c$-plane sapphire substrate with MOCVD, sample A represents a standard green LED structure. In preparing this sample, after the growth of a $30 \mathrm{~nm} \mathrm{GaN}$ buffer layer (grown at $535{ }^{\circ} \mathrm{C}$ ), a $3 \mu \mathrm{m} n$-GaN layer was grown at $1080{ }^{\circ} \mathrm{C}$ before the deposition of two periods of InGaN/GaN QW (3 $\mathrm{nm}$ in well thickness grown at $675^{\circ} \mathrm{C}, 12 \mathrm{~nm}$ in barrier thickness grown at $900{ }^{\circ} \mathrm{C}$ ). To complete the LED epitaxial structure, a $30 \mathrm{~nm} p-\mathrm{Al}_{0.2} \mathrm{Ga}_{0.8} \mathrm{~N}$ layer and then a $120 \mathrm{~nm}$ $p$-GaN layer were deposited on the top at the temperature of $945^{\circ} \mathrm{C}$. In sample B, an InGaN/GaN QW of $\sim 7 \%$ in indium content $\left(3 \mathrm{~nm}\right.$ well layer grown at $\left.760{ }^{\circ} \mathrm{C}\right)$ was added before growing the $12 \mathrm{~nm} \mathrm{GaN}$ bottom barrier of the first highindium QW, which has the same growth condition as that of sample A, to create the prestrain effect. The conditions for the rest part of epitaxial growth of sample B are the same as those of sample A. With the prestrain effect, the emission wavelength of sample B is shifted into the yellow range. To shift the emission wavelength back to the green range, the QW growth temperature was raised from 675 to $705^{\circ} \mathrm{C}$ to grow sample C. Except for the growth temperature for the high-indium QWs, all other growth conditions of sample C are the same as those of sample B. In this research, we are mainly concerned with the comparison between samples A (low QW growth temperature without the prestrain effect) and C (high QW growth temperature with the prestrain effect). For a more complete comparison, we also grew sample $\mathrm{D}$, in which the low-indium QW was removed to delete the prestrain effect while the high-indium QW growth temperature was maintained at the high level of $705{ }^{\circ} \mathrm{C}$. Without the prestrain effect, the emission wavelength of sample D falls into the blue range.

Figure 1 shows the XRD rocking curves of the four samples. Here, except for sample B, the oscillations of the secondary peaks on the left side of the GaN major peak can be clearly seen. Such oscillating secondary peaks are used for calibrating the average indium contents of the grown QWs with a fitting algorithm. The calibrated average indium contents of the QWs in sample A are 16.4\% and 16.3\% (from the top). Those of the three QWs in sample C are $16.7 \%$, $16.8 \%$, and $6.3 \%$. The $6.3 \%$ for the low-indium QW to create the prestrain effect is quite close to the designated 7\% level. The average indium contents of the two high-indium QWs in

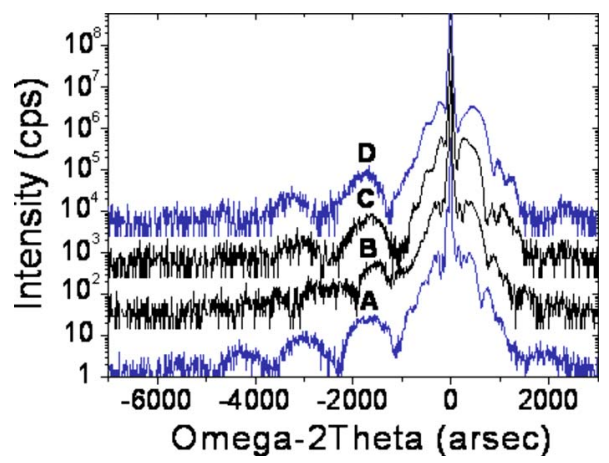

FIG. 1. (Color online) XRD rocking curves of the four samples.

sample $\mathrm{C}$ are very close to those of sample $\mathrm{A}$. The calibrated average indium contents of the QWs in sample D are $10.6 \%$ and $10.7 \%$, which are reasonable for blue emission.

\section{PHOTOLUMINESCENCE AND ELECTROLUMINESCENCE MEASUREMENTS}

Figure 2 shows the PL spectra, normalized to the peak level of sample $\mathrm{D}$, of the four samples at room temperature when $15 \mathrm{~mW}$ of pump laser power is applied to the sample. The PL measurement was excited by the $325 \mathrm{~nm}$ line of a $\mathrm{HeCd}$ laser. Because of the strong Fabry-Pérot oscillations in the PL spectra, it is difficult to identify the spectral peaks and hence the spectral center-of-mass (c.m.) values will be evaluated for representing the PL variation features throughout this paper. The spectral c.m. energies of samples A-D are $2.281,2.137,2.278$, and $2.682 \mathrm{eV}$, corresponding to 544 , 580,544 , and $462 \mathrm{~nm}$ in wavelength, respectively, as listed in Table I. One can see that the blue (sample D) and yellow (sample B) emissions are the strongest and weakest, respectively. In particular, the PL intensity of sample C is significantly stronger than that of sample A, showing the improvement of emission efficiency by using the prestrain effect. The ratios of integrated PL intensity at room temperature of the four samples are also shown in Table I. Here, one can see the PL enhancement of about 2.4 times by applying the prestrain technique to the growth of green-emitting QWs.

Figure 3 shows the EL spectra of the four samples at 20 $\mathrm{mA}$ in injection current, normalized to the peak level of sample D. The EL data were obtained by the quick test through indium-ball contacts on a 2 in. epitaxial wafer. The comparison of such data can avoid the complicated process factors in fabricating LED. The EL spectral peak positions of

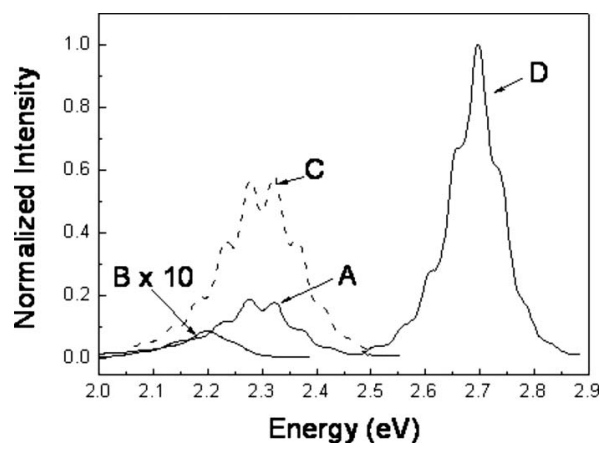

FIG. 2. Normalized PL spectra of the four samples at room temperature. 
TABLE I. Comparisons of various parameters between the four samples.

\begin{tabular}{|c|c|c|c|c|}
\hline Sample & A & $\mathrm{B}$ & $\mathrm{C}$ & $\mathrm{D}$ \\
\hline PL spectral c.m. at room temperature $(\mathrm{eV} / \mathrm{nm})$ & $2.281 / 544$ & $2.137 / 580$ & $2.278 / 544$ & $2.682 / 462$ \\
\hline EL spectral peak at $20 \mathrm{~mA}(\mathrm{eV} / \mathrm{nm})$ & $2.366 / 524$ & $2.250 / 551$ & $2.353 / 527$ & $2.690 / 461$ \\
\hline Integrated PL intensity ratio at room temperature & 1 & 0.09 & 2.40 & 2.85 \\
\hline Integrated EL Intensity ratio at $20 \mathrm{~mA}$ & 1 & 0.08 & 2.82 & 4.17 \\
\hline $\mathrm{IQE}(\%)$ & 5.7 & 1.8 & 15.1 & 22.7 \\
\hline PL spectral c.m. shift (meV) & 14.5 & 17.5 & 8.9 & 3.8 \\
\hline EL spectral peak shift (meV) & 45.6 & 78.8 & 24.7 & 20.8 \\
\hline$\alpha$ & 3.2 & 3.5 & 0.5 & 1.2 \\
\hline$E_{A 1}(\mathrm{meV})$ & 16.0 & 12.8 & 7.6 & 22.3 \\
\hline$\beta$ & 410.0 & 1904.0 & 26.2 & 45.0 \\
\hline$E_{A 2}(\mathrm{meV})$ & 84.7 & 100.6 & 43.8 & 70.2 \\
\hline PL decay time (ns) & 30.1 & $\cdots$ & 32.9 & 19.1 \\
\hline XRD calibrated average indium content $(\%)$ & 16.4 (top)/16.3 & $\cdots$ & 16.7 (top)/16.8/6.3 & 10.6 (top) $/ 10.7$ \\
\hline SSA calibrated average indium content $x_{a}(\%)$ & 15.6 (top)/15.2 & 18.3 (top)/18.6/6.9 & 15.5 (top)/15.7/7.1 & 9.1 (top)/9.5 \\
\hline SSA calibrated indium composition variation $\Delta x(\%)$ & 17.5 (top)/23.6 & 16.1 (top)/22.7 & 11.6 (top) $/ 15.8$ & 10.5 (top)/12.0 \\
\hline
\end{tabular}

samples A-D are located around 2.366, 2.250, 2.353, and $2.690 \mathrm{eV}$ (corresponding to 524, 551, 527, and $461 \mathrm{~nm}$ in wavelength), respectively, which are somewhat higher than those of PL spectra. Such blueshifts of EL spectra, relative to the PL spectra, are attributed to the screening of the quantum-confined Stark effect (QCSE) as to be discussed later. Their relative intensities are also similar to those of PL measurement. Their ratios are listed in Table I to show the 2.82 times increase in using the prestrain effect for green emission. In Table I, one can see that the EL intensity ratios of samples $\mathrm{C}$ and D are higher than their counterparts in the PL measurement. In the steady state, the estimated carrier density in the QWs in the EL measurement is in the order of $8.1 \times 10^{17} \mathrm{~cm}^{-3}$ at the injection current level of $20 \mathrm{~mA}$ if all the supplied carriers are completely injected into the emitting QWs. On the other hand, at the pump power of $15 \mathrm{~mW}$, the photogenerated carrier density in the QWs in the PL measurement is in the order of $3 \times 10^{17} \mathrm{~cm}^{-3}$ depending on the absorption coefficient of the InGaN in the QW. The more significant enhancements of EL intensity in samples C and D can be due to the higher carrier densities supplied to the QWs. The other possible cause is that in the EL measurement, with the applied voltage, carrier injection into the QWs can be more efficient. Nevertheless, it is difficult to draw any conclusion because many factors can be involved, including

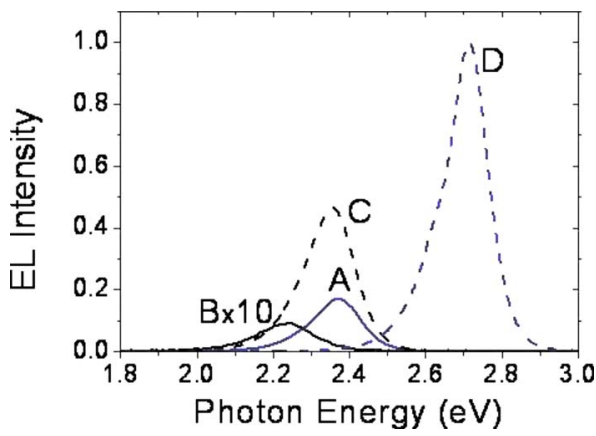

FIG. 3. (Color online) Normalized EL spectra of the four samples at the injection current of $20 \mathrm{~mA}$. current leakage, material defect density, device resistance, material absorption coefficient, carrier diffusion constant, and piezoelectric field.

Figure 4 shows the temperature dependencies of the integrated PL intensity of the four samples under the same measurement condition as that for Fig. 2. The PL intensities drop with increasing temperature due to the increasing capture probability of carriers by nonradiative recombination centers. The ratio of the room-temperature level over that at $10 \mathrm{~K}$ is usually interpreted as the IQE. Although such an interpretation is not necessarily precise, the IQE defined here can provide us with a reliable trend in comparing the densities of nonradiative recombination center in the four samples. The IQE values of samples A-D are 5.7\%, 1.8\%, 15.1\%, and $22.7 \%$, respectively, as also shown in Table I. The relatively higher and lower IQEs in the blue and yellow ranges, respectively, have been widely reported. The comparison between samples A and C shows an $\sim 167 \%$ increase in IQE in using the prestrained growth technique for fabricating greenemitting QWs.

Figure 5 shows the PL spectral features of the four samples as functions of pump power. In increasing the PL pump power from 3 to $15 \mathrm{~mW}$, the blueshifts of the c.m. energies of samples A-D are 14.5, 17.5, 8.9, and $3.8 \mathrm{meV}$, respectively. The blueshift range of PL feature in pump-

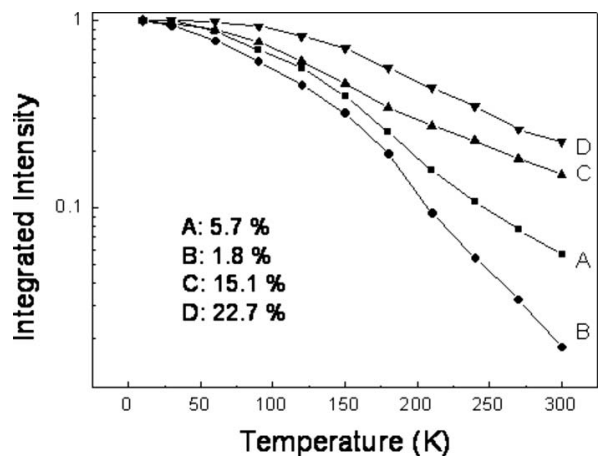

FIG. 4. Temperature-dependent integrated PL intensities of the four samples showing their IQEs. 


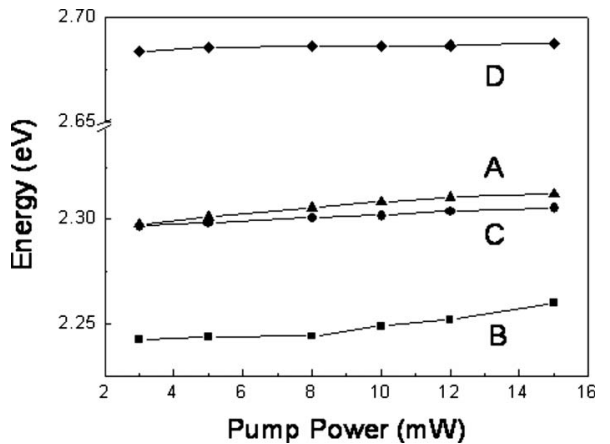

FIG. 5. Pump-power dependent PL spectral c.m.-energy variations of the four samples.

power dependent PL measurement is usually used as an important indicator of the strength of the QCSE. ${ }^{16}$ The QCSE exists in an InGaN/GaN QW because of its strong piezoelectric field. This effect means the $\mathrm{QW}$ potential tilt, leading to the reduction in radiative recombination rate and the emission redshift. When carriers are supplied into the $\mathrm{QW}$, the field-screening effect results in enhanced emission efficiency and spectral blueshift. Normally, a QW of higher indium content and hence longer-wavelength emission has a stronger QCSE because of the larger lattice mismatch between the well and barrier layers. The blueshift ranges of c.m. energy in Fig. 5 show the relatively larger and smaller shifts in the yellow- (B) and blue-emitting (D) samples, respectively. For the two green-emitting samples of almost the same emitting spectral feature, the one of prestrained growth (sample C) has a smaller shift range of $8.9 \mathrm{meV}$, compared to the range of $14.5 \mathrm{meV}$ of sample A. In other words, with the prestrained growth, the QCSE is reduced.

The similar spectral variation trend can also be observed by injecting different current levels into the four LED epitaxial samples. Figure 6 shows the variations of EL spectral peak positions of the four samples when the injection current increases from 5 to $50 \mathrm{~mA}$. The spectral blueshift ranges of samples A-D are 45.6, 78.8, 24.7, and $20.8 \mathrm{meV}$, respectively. Again, the relatively larger and smaller spectral blueshifts in the samples of higher and lower indium contents, respectively, can be seen. By comparing samples A and C, one can see the smaller blueshift in the prestrained sample (sample C). It is noted that in the measurement of EL spectral peak position variation, the Joule heating of the sample is supposed to produce a redshift trend of the EL spectrum.

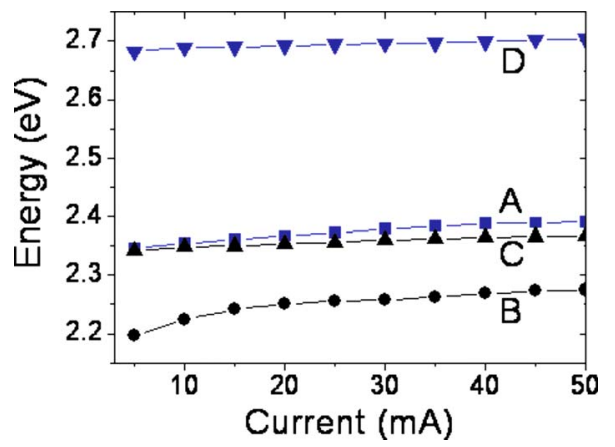

FIG. 6. (Color online) Injection-current dependent EL spectral peak-energy variations of the four samples.

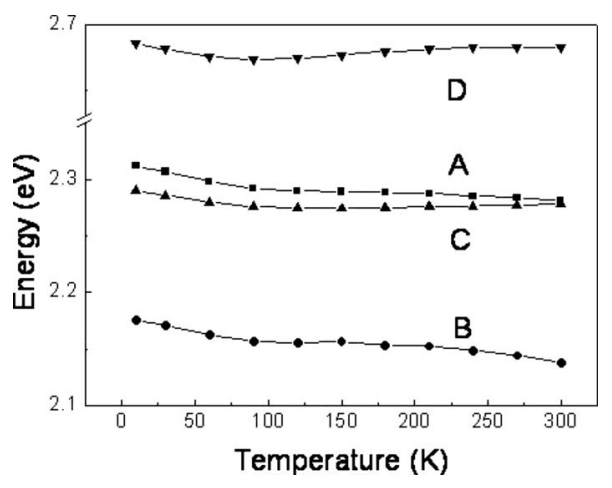

FIG. 7. Temperature-dependent PL spectral c.m.-energy variations of the four samples.

Therefore, the heating effect can partially compensate the blueshift trend, which is due to the QCSE screening. In other words, in a sample of a stronger heating effect (higher resistance), the QCSE-screening-induced blueshift range can be underestimated. The measured turn-on voltages of samples $\mathrm{A}-\mathrm{D}$ are $2.9,4.1,3.0$, and $3.7 \mathrm{~V}$, respectively. The series resistance levels of samples A-D are 107.3, 265.3, 81.2, and $128.2 \Omega$, respectively. Therefore, the heating effect is the strongest in sample B, followed by samples D, A, and then C. The prestrained green LED sample (sample C) has the weakest heating effect. Therefore, as shown in Table I, when compared with sample $\mathrm{C}$, the relatively larger EL spectral blueshift ranges in increasing injection current of samples A and B are actually underestimated. This trend manifests the even relatively weaker QCSE in sample C when compared with samples A and B. Regarding sample D, its larger turn-on voltage and series resistance can be due to the poor contacts we fabricated. Its QCSE-screening-induced blueshift range can be underestimated. However, this does not affect the conclusions drawn here.

\section{DISCUSSIONS}

To understand the causes for the enhanced emission efficiency and reduced blueshift in increasing the carrier density in the QW, we first demonstrate the temperaturedependent PL c.m. variations of the four samples in Fig. 7. One can see that in each of the four samples, the c.m. energy shows an $\mathrm{S}$-shape variation as a function of temperature. In other words, in each sample, the spectral c.m. redshifts first, then blueshifts, and finally redshifts again as temperature increases from 10 to $300 \mathrm{~K}$. Such a behavior is usually regarded as an indicator of significant carrier localization in a QW. ${ }^{17}$ The carrier localization behavior is due to the formation of indium-rich clusters and composition fluctuations in a QW. ${ }^{18}$ With the carrier localization behavior, carriers can be confined in potential minima, away from nonradiative recombination centers, such that the emission efficiency can usually be enhanced. The results shown in Fig. 7 indicate that all the four samples have significant carrier localization behaviors. However, from the c.m. variations, it is difficult to judge the differences in the degree of carrier localization.

The degree of carrier localization can usually be determined by plotting and fitting an Arrhenius curve, ${ }^{19}$ as demonstrated in Fig. 8. Here, the normalized PL intensity of each 


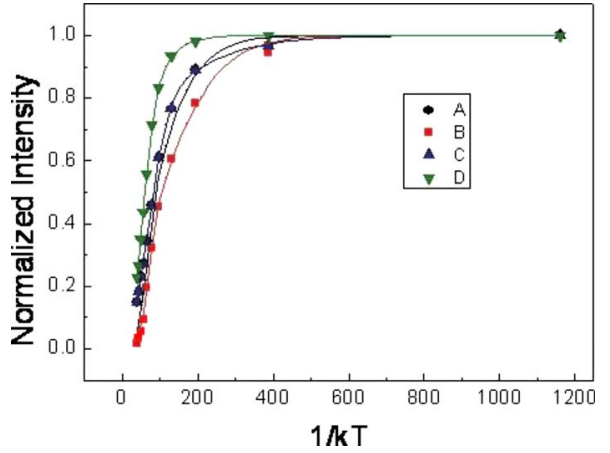

FIG. 8. (Color online) Arrhenius plots of the four samples. The fitting curves are used to calibrate the parameters in Eq. (1).

sample is plotted as a function of $1 / k T$ with $k$ and $T$ representing the Boltzmann constant and absolute temperature, respectively. The data points are then fitted with a curve as

$$
I(T)=\left[1+\alpha \exp \left(-E_{A 1} / k T\right)+\beta \exp \left(-E_{A 2} / k T\right)\right]^{-1} .
$$

Here, $I(T)$ represents the normalized integrated PL intensity as a function of $T$. The symbols $\alpha$ and $\beta$ are two parameters related to the density of nonradiative recombination center in the sample. They can also be interpreted as an indicator for the degree of carrier localization. When the density of nonradiative recombination center is low and/or the carrier localization behavior is strong, $\alpha$ and $\beta$ are expected to be small and vice versa. In Eq. (1), $E_{A 1}$ and $E_{A 2}$ are two energies, called the activation energies, describing the potential barrier carriers need to overcome before they are annihilated at nonradiative recombination centers. Therefore, the values of $E_{A 1}$ and $E_{A 2}$ are related to the spatial potential distribution in a $\mathrm{QW}$, including the potential barrier heights of carrier localization. The two exponential components of $E_{A 1}$ and $E_{A 2}$ in Eq. (1) are designed to describe the properties at the lowand high-temperature ranges, respectively. They correspond to two groups of potential minimum of different barrier potential heights. A lower potential barrier $\left(E_{A 1}\right)$ can be overcome by the carrier thermal energy in the low-temperature range (below $\sim 100 \mathrm{~K}$ ) However, a high potential barrier $\left(E_{A 2}\right)$ can be overcome by the carrier thermal energy only in the high-temperature range.

The parameters for plotting the fitting curves in Fig. 8 are listed in Table I. Generally, with the high (low) indium content of sample B (D), the $\alpha$ and $\beta$ values are relatively larger (smaller). However, no clear trend can be observed in the comparison of the activation energies. Nevertheless, between samples $\mathrm{A}$ and $\mathrm{C}$, one can see the systematical trends that all the $\alpha, \beta, E_{A 1}$, and $E_{A 2}$ values are smaller in sample $\mathrm{C}$. These comparison results indicate that in the prestrained growth sample $(\mathrm{C})$, the defect density is lower and the carrier localization effect is weaker when compared with the control sample (A).

Another approach for understanding the carrier recombination mechanism is the measurement of TRPL. Figure 9 shows the PL intensity decay profiles of samples A, C, and D from the TRPL measurement. The TRPL measurement was excited by the second-harmonic (390 nm in wavelength) of a femtosecond Ti:sapphire laser $(\sim 77 \mathrm{MHz}$ in pulse repetition

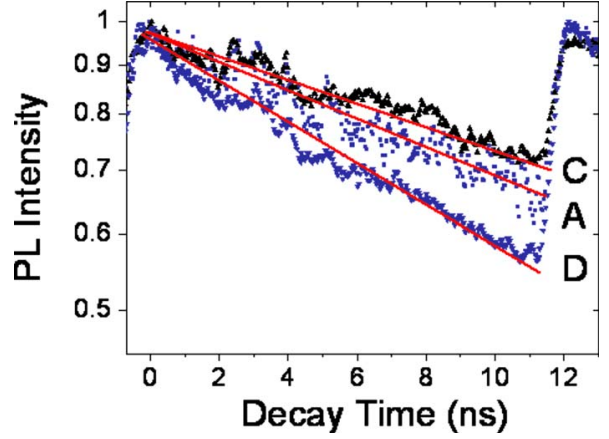

FIG. 9. (Color online) Decay profiles of TRPL intensities of samples A, C, and $\mathrm{D}$ at room temperature. The fitting lines are plotted for calibrating the exponential decay times.

rate) and recorded by a streak camera. The TRPL result of sample B is missing because its PL intensity is not sufficiently strong to be monitored by the used streak camera. The fitting lines for calibrating the exponential decay time constants are also shown in Fig. 9. It is noted that because the pulse repetition period $(\sim 12 \mathrm{~ns})$ is significantly shorter than the measured decay time, the TRPL intensity does not decay to its zero level before the next excitation pulse is applied to the sample. Therefore, periodical peak features with a period of $\sim 12$ ns can be seen in Fig. 9. However, this periodical behavior does not affect the calibration of PL decay time. Suppose $A$ is the peak intensity of the timeresolved profile generated by single-pulse excitation, $\tau_{d}$ is the decay time constant, and $\tau_{p}$ is the excitation pulse repetition period, with the first pulse excitation set at $t=0$, the TRPL intensity profile under multiple-pulse excitation is given by an infinite series as

$$
\begin{aligned}
A e^{-t / \tau_{d}} & +A e^{-\tau_{p} / \tau_{d}} e^{-t / \tau_{d}}+A e^{-2 \tau_{p} / \tau_{d}} e^{-t / \tau_{d}}+\cdots=A(1 \\
& \left.+e^{-\tau_{p} / \tau_{d}}+e^{-2 \tau_{p} / \tau_{d}}+\cdots\right) e^{-t / \tau_{d}}=\frac{A}{1-e^{-\tau_{p} / \tau_{d}}} e^{-t / \tau_{d}} .
\end{aligned}
$$

As shown in the last expression of Eq. (2), the PL intensity decays exponentially with the time constant $\tau_{d}$ within a period of pulse excitation. Therefore, by calibrating the exponential decay constant in a curve shown in Fig. 9, we can obtain a reasonable PL decay time constant. The decay profiles shown in Fig. 9 have been normalized such that their peak intensities are unity. Such a normalization procedure does not affect the calibration of decay time constant. The decay time constants are listed in Table I to show 30.1, 32.9, and $19.1 \mathrm{~ns}$ for samples A, C, and D, respectively. The longer decay time constants of the green-emitting samples (A and C) when compared with the blue-emitting one (D) can be attributed to the relatively stronger QCSE. ${ }^{20}$ Such an effect is expected to be stronger in a QW of higher indium content. In comparing sample $\mathrm{A}$ with $\mathrm{C}$, the slightly longer decay time in the prestrained sample cannot be attributed to stronger QCSE because we have shown the relatively weaker QCSE in this sample from Figs. 5 and 6 . The significantly higher IQE of sample C (2.7 times that of sample A) implies that the slightly longer decay time can be attributed to the lower defect density because of the higher QW growth temperature. Nevertheless, it is believed that the QCSE is still the 


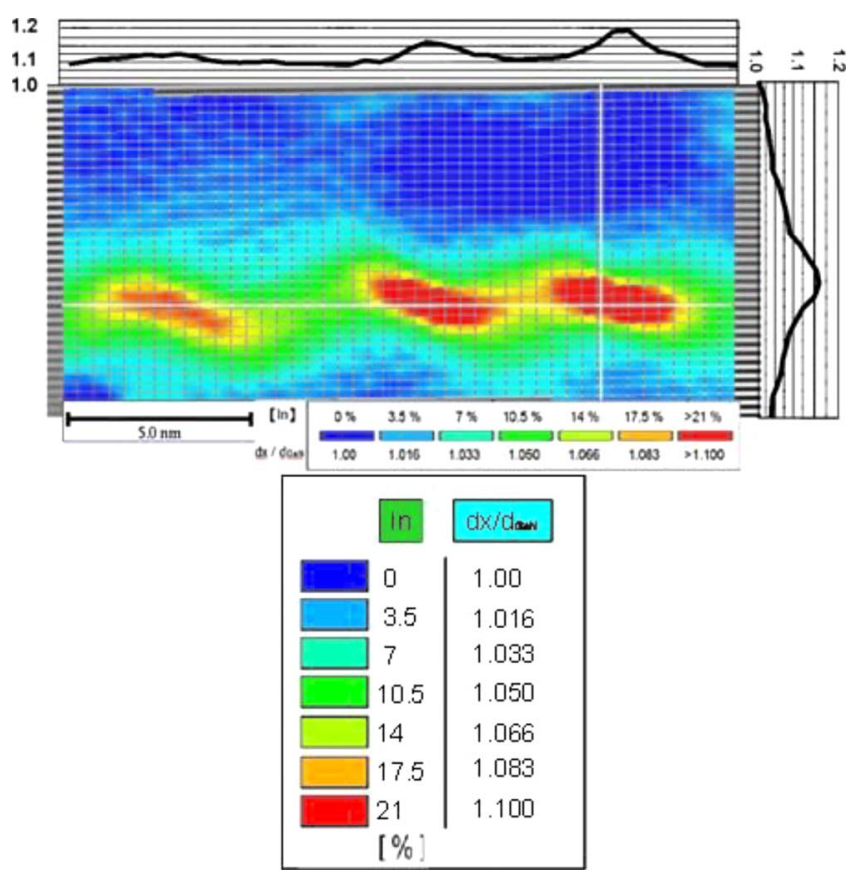

FIG. 10. (Color online) SSA image of the second QW from the top of sample A. The color coding legend is shown in the lower portion of the figure.

dominating factor in sample $\mathrm{C}$ to control the emission behavior. In this sample, the relatively weaker QCSE and carrier localization when compared with sample A are supposed to shorten the decay time. However, the significantly lower defect density can result in an effect to cancel the shortening trend and slightly elongate the decay time.

TEM images are quite useful in understanding the nanostructures of the used samples and hence in further understanding their emission mechanisms. We have performed high-resolution TEM study by using the strain state analysis (SSA) algorithm to map the strain distribution near each QW. ${ }^{21,22}$ From the strain mapping, we can calibrate the composition distribution near each QW. Figures 10 and 11 show the SSA images of the second QWs (counted from the top) of samples A and C, respectively. The color coding legend is shown below the SSA image in Fig. 10. The images show the distributions of $d x / d_{\mathrm{GaN}}$, which correspond to various indium

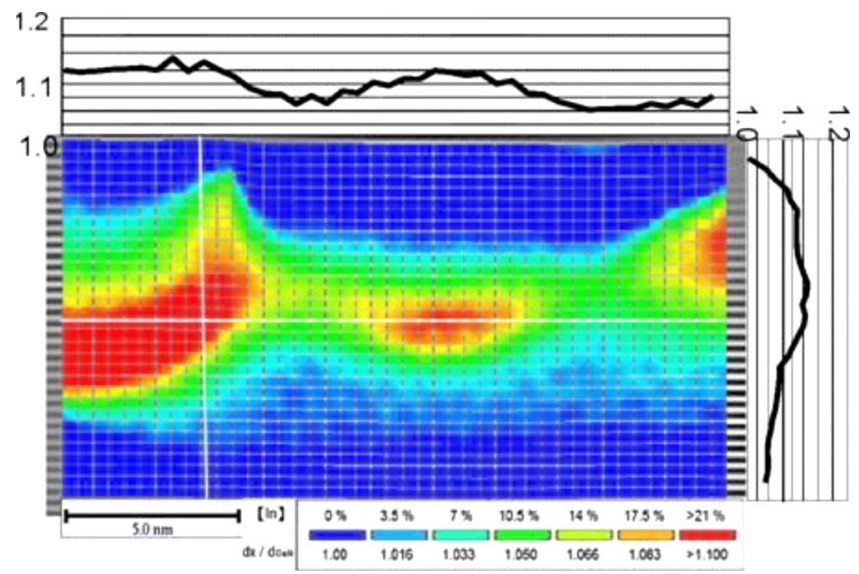

FIG. 11. (Color online) SSA image of the second QW from the top of sample $\mathrm{C}$. compositions as shown in the legend. Here, $d_{\mathrm{GaN}}$ represents the lattice constant of $\mathrm{GaN}$ and $d x$ stands for the calibrated local lattice size. ${ }^{10}$ The ratio $d x / d_{\mathrm{GaN}}$ describes the local relative lattice size and hence corresponds to the local indium composition, as shown in the legend of Fig. 10. In the upper and right ends of Figs. 10 and 11, a horizontal and a vertical line-scan $d x / d_{\text {GaN }}$ value are plotted. From the line-scan results, one can estimate the degree of indium composition fluctuation in a QW by evaluating the difference between the absolute maximum and minimum levels in a horizontal linescan profile, which is defined as $\Delta x$. Also, the average indium content $x_{a}$ of a QW can be estimated by integrating the indium composition distribution near the designated $\mathrm{QW}$ area. Based on this approach, we obtain the $x_{a}$ and $\Delta x$ values of all those QWs in the four samples, as listed in Table I. Here, one can see that in samples A and C, the average indium contents of high-indium QWs range between $15 \%$ and $16 \%$, which are reasonably consistent with the levels of $16 \%-17 \%$ obtained by XRD fitting (also listed in Table I). The value of $7.1 \%$ for the low-indium QW in sample $\mathrm{C}$ is also reasonably consistent with the level of $6.3 \%$ from the XRD measurement. The $x_{a}$ values of the high-indium QWs in samples B and $\mathrm{D}$, which are in the ranges of $18 \%-19 \%$ and $9 \%-10 \%$, respectively, are also quite reasonable for emitting yellow and blue lights. In Table I, we also show the $\Delta x$ values of the high-indium QWs. Here, one can see that in either the top or the bottom QW, the composition fluctuation of sample $\mathrm{A}$ is always larger than that of sample $\mathrm{C}$, indicating that the carrier localization effect in sample $\mathrm{C}$ can be weaker than that in sample A. This trend is consistent with the calibration results of $E_{A 1}$ and $E_{A 2}$ in the Arrhenius plots. It is noted that because TEM images are obtained only in small regions of the sample, the SSA data cannot be used as the single evidence for drawing a conclusion. Here, the calibrated data of average indium content and indium composition variation are used only for showing the consistent trends, not used as the crucial supports for drawing the conclusion. Because it is difficult to define a parameter for more suitably representing the composition fluctuation, we simply evaluated the valley-to-crest difference along a line scan, which can show the largest contrast. Regarding the systematically smaller composition variations in the top QWs, when compared with those of the second QWs, in all the four samples, although we have observed a similar trend in some other samples of similar structures in previous studies, it is difficult to make any conclusion because of the reason given above. This issue deserves further investigation. With all the arguments above, the results shown in Figs. 10 and 11 and Table I can still provide us with a consistent trend with that from the Arrhenius plots.

Based on the data and discussions above, one can conclude that the higher emission efficiency of sample $\mathrm{C}$ when compared with sample A is mainly due to the lower defect density because of the higher QW growth temperature. Also, with the prestrained growth, both the QCSE and the carrier localization effect become weaker. The weaker QCSE in sample $\mathrm{C}$ can be regarded as another advantage of using the prestained growth technique. It is noted that in one of our previous publications, ${ }^{23}$ we attributed the reduced blueshift 
range in increasing the injection current of a LED fabricated from a prestrained growth epitaxial structure (even though the QCSE was shown to become stronger) to the nonuniform indium content distribution among the five designated emitting QWs. ${ }^{23}$ However, in the samples of this paper, only two emitting QWs were prepared and the QCSE was shown to become weaker in the prestrained growth sample based on the pump-power dependent PL measurement. Therefore, it is believed that the major cause for reduced EL spectral shift in sample $\mathrm{C}$ of this paper is the reduced QCSE. In the previous study, the prestrained growth led to significantly higher indium content when compared with the control sample. In this situation, the significantly higher indium content resulted in enhanced QCSE even though the prestrained growth may somewhat reduce this effect. In the previous study, we compared the prestrained sample with a control sample of lower indium content and a shorter emission wavelength. However, in the current research, we compare the prestrained and control samples of about the same indium content and about the same emission wavelength.

\section{CONCLUSIONS}

In summary, we have demonstrated the enhanced emission efficiency and reduced spectral shifts of a green InGaN/ GaN QW LED epitaxial structure by using the prestrained MOCVD growth technique when compared with a control sample of about the same indium content and emission wavelength with conventional growth. By adding an $\sim 7 \%$-indium $\mathrm{InGaN} / \mathrm{GaN} \mathrm{QW}$ to the structure before the growth of designated emitting high-indium QWs, the growth temperature of the emitting QWs could be raised by $30{ }^{\circ} \mathrm{C}$ while keeping about the same emission wavelength around $544 \mathrm{~nm}$ in PL and $525 \mathrm{~nm}$ in EL. With the increased growth temperature, the crystal quality was improved and the emission efficiency became higher. The IQE, room-temperature PL intensity, and EL intensity at $20 \mathrm{~mA}$ were increased by $\sim 167 \%, \sim 140 \%$, and $\sim 182 \%$, respectively. Also, the spectral blueshift in increasing injection current in the range of $45 \mathrm{~mA}$ was decreased by $46 \%$. Based on the pump-power dependent PL measurement, it was concluded that the QCSE became weaker under prestrained growth. Also, from the calibration of the Arrhenius plots and the SSA of TEM study, the carrier localization effect was shown to become weaker in the prestrained growth sample. Therefore, the enhanced emission efficiency could be attributed to the decreased defect density in the prestrained sample that was also confirmed by the calibration of the Arrhenius plots. Nevertheless, in the situation of maintaining the same average indium content, the strain condition under the prestrained growth for simultaneously reducing the QCSE and indium-rich clustering effect deserves further investigation.

\section{ACKNOWLEDGMENTS}

This research was supported by the National Science Council, Republic of China under Grant Nos. NSC 96-2120M-002-008 and NSC 96-2628-E-002-044-MY3, by the U.S. Air Force Scientific Research Office under Contract No. AOARD-07-4010, and by the Excellent Research Projects of National Taiwan University under Grant No. 97R0061-04.

${ }^{1}$ T. Gessmann and E. F. Schubert, J. Appl. Phys. 95, 2203 (2004).

${ }^{2}$ Y. S. Lin, K. J. Ma, C. Hsu, S. W. Feng, Y. C. Cheng, C. C. Liao, C. C. Yang, C. C. Chuo, C. M. Lee, and J. I. Chyi, Appl. Phys. Lett. 77, 2988 (2000).

${ }^{3}$ S. W. Feng, E. C. Lin, T. Y. Tang, Y. C. Cheng, H. C. Wang, C. C. Yang, K. J. Ma, C. H. Shen, L. C. Chen, K. H. Kim, J. Y. Lin, and H. X. Jiang, Appl. Phys. Lett. 83, 3906 (2003).

${ }^{4}$ I. K. Park, M. K. Kwon, J. O. Kim, S. B. Seo, J. Y. Kim, J. H. Lim, S. J. Park, and Y. S. Kim, Appl. Phys. Lett. 91, 133105 (2007).

${ }^{5}$ Y. H. Cho, Y. P. Sun, H. M. Kim, T. W. Kang, E.-K. Suh, H. J. Lee, R. J. Choi, and Y. B. Hahn, Appl. Phys. Lett. 90, 011912 (2007).

${ }^{6}$ Y. S. Lin, K. J. Ma, C. C. Yang, and T. E. Weirich, J. Cryst. Growth 242, 35 (2002).

${ }^{7}$ J. B. Limb, W. Lee, J. H. Ryou, D. Yoo, and R. D. Dupuis, J. Electron. Mater. 36, 426 (2007).

${ }^{8}$ J.-H. Ryou, W. Lee, J. Limb, D. Yoo, J. P. Liu, R. D. Dupuis, Z. H. Wu, A. M. Fischer, and F. A. Ponce, Appl. Phys. Lett. 92, 101113 (2008).

${ }^{9}$ C. F. Huang, T. Y. Tang, J. J. Huang, W. Y. Shiao, C. C. Yang, C. W. Hsu, and L. C. Chen, Appl. Phys. Lett. 89, 051913 (2006).

${ }^{10}$ Y. S. Chen, L. J. Yao, Y. L. Lin, L. Hung, C. F. Huang, T. Y. Tang, J. J. Huang, W. Y. Shiao, and C. C. Yang, J. Cryst. Growth 297, 66 (2006).

${ }^{11}$ W. Y. Shiao, C. F. Huang, T. Y. Tang, J. J. Huang, and C. C. Yang, J. Appl. Phys. 101, 113503 (2007).

${ }^{12}$ I.-h. Ho and G. B. Stringfellow, Appl. Phys. Lett. 69, 2701 (1996).

${ }^{13}$ L. K. Teles, J. Furthmuller, L. M. R. Scolfaro, J. R. Leite, and F. Bechstedt, Phys. Rev. B 62, 2475 (2000).

${ }^{14}$ H. S. Chen, C. F. Lu, D. M. Yeh, C. F. Huang, J. J. Huang, and C. C. Yang, IEEE Photonics Technol. Lett. 18, 2269 (2006).

${ }^{15}$ C. F. Huang, T. Y. Tang, J. J. Huang, and C. C. Yang, Appl. Phys. Lett. 90, 151122 (2007).

${ }^{16}$ T. Takeuchi, S. Sota, M. Katsuragawa, M. Komori, H. Takeuchi, H. Amano, and I. Akasaki, Jpn. J. Appl. Phys., Part 2 36, L382 (1997).

${ }^{17}$ Y. H. Cho, G. H. Gainer, A. J. Fischer, J. J. Song, S. Keller, U. K. Mishra, and S. P. DenBaars, Appl. Phys. Lett. 73, 1370 (1998).

${ }^{18}$ P. Ruterana, S. Kret, A. Vivet, G. Maciejewski, and P. Dluzewski, J. Appl. Phys. 91, 8979 (2002).

${ }^{19}$ R. Seitz, C. Gaspar, T. Monteiro, E. Pereira, M. Leroux, B. Beaumont, and P. Gibart, J. Cryst. Growth 189-190, 546 (1998).

${ }^{20}$ S. F. Chichibu, A. C. Abare, M. S. Minsky, S. Keller, S. B. Fleischer, J. E. Bowers, E. Hu, U. K. Mishra, L. A. Coldren, S. P. DenBaars, and T. Sota, Appl. Phys. Lett. 73, 2006 (1998).

${ }^{21}$ A. Rosenauer, U. Fischer, D. Gerthsen, and A. Förster, Appl. Phys. Lett. 71, 3868 (1997).

${ }^{22}$ D. Gerthsen, E. Hahn, B. Neubauer, V. Potin, A. Rosenauer, and M. Schowalter, Phys. Status Solidi C 0, 1668 (2003).

${ }^{23}$ C. F. Huang, C. Y. Chen, C. F. Lu, and C. C. Yang, Appl. Phys. Lett. 91, 051121 (2007). 